\title{
Healthcare associated vancomycin- resistant enterococci
}

\author{
Murtaza Mustafa, Muhammad Iftikhar, M Yusof Ibrahim, Rajesh K Muniandy \\ Faculty of Medicine and Health Sciences, University Malaysia Sabah, KotaKinabalu, Sabah Malaysia.
}

\begin{abstract}
Vancomycin resistant enterococci (VRE) were first detected in the 1980s, since then VRE infections are reported worldwide. VRE can be isolated from healthy people, hospital environment and from farmed chicken.VRE infections are more severe, increased mortality and economic burden. Healthcare associated infections are mainly due to antibiotic therapy, patient characteristics, colonization pressure and exposure to contaminated environment. VRE are resistant to most commonly used antibiotics, with the ability to acquire resistance to other available antimicrobials and to transfer antibiotic resistance to other gut flora. CDC Hospital Infection Control Practices Advisory Committee (HICPAC) recommendations are useful to minimize transmission of VRE with multidisciplinary approach, and restricted cephalosporin usage has been associated with decreased VRE transmission in hospitals.
\end{abstract}

KEY WORDS: Vancomycin, Enterococcus facecium.Resistancemechanisms, VRE control

\section{INTRODUCTION}

Vancomycin -resistant Enterococcus faecalis and Enterococcus facecium clinical isolates were first documents in Europe in the late 1980s [1,2].Since then ,VRE have been associated with outbreaks of hospital acquired (nosocomial) infections around the world. In the United States from 2006 to 2007, 4\% of vancomycinresistant E.faceium was healthcare- infections [3].VRE can be carried by healthy people who come into contact with the bacteria. The most likely place where such contact can occur is in the hospital although it is thought that a significant percentage of intensively farmed chicken also carry VRE [4,5].Compared with vancomycin susceptible enterococci(VSE),VRE infections cause more severe disease, increased mortality and have significant additional economic burden. Antibiotic treatment options for VRE are limited and pan-resistance can occur [6].Enterocooci with acquired transferable high level resistance to vancomycin were first detected in feces from two leukemia patients from Europe in 1986 [7].Hospital outbreaks were increasingly described over the next decade across Europe and the USA. Internationally, the important the important resistance genotypes of VRE have been either vanA or vanBoperons usually carried by Enterococcus facium in a transposon located within a large transferable plasmid. There is evidence of global spread of clonal complex of hospital-associated ampicillin resistant E.faecium (CCI7) that includes both VSE and VRE (either vanA or vanB) and has a number of putative virulence factors for hospital adaption and spread [8,9].Researchers in Japan reported patients infected or colonized with Enterococcus faceiumvanB [10].In Australia,vanB-.E,faceium has predominated since late 1990s.A 2010 Australian VRE survey indicated increasing prevalence of VRE vanBE.faceium from the CC17 in a number of States [11].Risk factors for healthcare-associated colonization and or infections with VRE have been identified, they include: (a)previous antibiotic exposure,(b)patient characteristics,(c)colonization pressure,(d)exposure to contaminated surfaces[12].Control measures of VRE include the enhanced standard precautions(horizontal measures) in healthcare that reduce infections caused by all organisms, not just VRE. Effective antimicrobial stewardship programs are paramount and should target reduction in the use of extendedspectrum cephalosporin's, carbapenems and fluoroquinolones[13].This paper reviews mechanisms of resistance, epidemiology, and control of VRE.

\section{MECHANISMS OF RESISTANCE}

Among enterococci seven types of glycopeptide resistance have been described (Vana,VanB,VanC,VanD,VanEVanG, and Van I) which are based on their specific ligase genes (e.g.,vanA,vanB, etc).Glycopeptide resistance in enterococci is classified as either intrinsic( as a species characteristic) or acquired. The former is a characteristic of the motile species Enterococcus gallinarum and Enterococcus casseliflavus, these strains carry vanC-1 and vanC-2/vanC-3genes. Acquired resistance is found most often in E.faceium followed by E.faecalis, and is much less common in other enterococcal strains [14 15]. 


\section{Resistance to $\beta$-lactam}

Complete or relative resistance to $\beta$-lactams is a characteristics features of the genus Enterococcus.E.facecalis is typically 10 to 100 times less susceptible to penicillin than other streptococci, whileE.facecium is at least 4 to 16 times less susceptible than E.faecalis [16]. While most isolates of E.faecalis are inhibited by concentrations of penicillin or ampicillin (1 to $8 \mu \mathrm{g} / \mathrm{ml})$ easily achievable in humans, isolates of E.faecium usually require an average of 16 to $64 \mu \mathrm{g} / \mathrm{ml}$ to inhibit growth , although some isolates are more resistant [17].An additional problem with enterococci is that they are typically tolerant to $\beta$-lactams (i.e $. \mathrm{MBC} / \mathrm{MIC}$ of $>32$ ). The major mechanism underlying this resistance has the production of low-affinity PBP [18].Fontana et al. showed that loss of the ability of a strain of E.facecium to produce PBP5 caused this highly penicillin- resistant strain to become hyper susceptible to penicillin[19]. $\beta$-lactamase production is inducible, $\beta$ lactamase production in enterococci is constitutive low level, and inoculum dependent[20].

\section{Resistance to Aminoglycoside}

Early researchers demonstrated that two types of streptomycin resistance occur in enterococci :(i) moderate level resistance (MIC,62,to $500 \mu \mathrm{g} / \mathrm{ml}$ ), because of low permeability, which can overcome with penicillin ( which increases the cellular uptake of the aminoglycoside);and (ii) high level resistance (MIC, $\geq 2,000 \mu \mathrm{g} / \mathrm{ml}$ ), which is either ribosomally mediated or due to the production aminoglycoside-inactivating enzymes [21].Since enterococcal resistance to gentamicin and streptomycin occur by different mechanisms, it is important to test susceptibilities to both agents. Gentamicin resistance is predominantly the result of the presence of the inactivating enzyme2"-phosphotransferase-6'-acetylytransferase conferring resistance to gentamicin, tobramycin ,netlimicin,amikacin and kanamycin. Hence gentamicin resistance is a good predictor of resistance to other aminoglycosides except streptomycin is encountered mainly in enterococcal strains that produce streptomycin adenyltransferase; these strains remain susceptible to gentamicin [20,rept ].

\section{Resistance to Vancomycin}

There are five recognized phenotypes of vancomycinresistance, VanA,VanB, VanC,VanD, and $\operatorname{VanE}[22]$.Two of these (VanA and $V a n B$ ) are mediated by newly acquired gene clusters not previously found in enterococci.VanA and VanB resistance phenotypes were mainly described primarily in E.faecalis and E.faceium.VanA-resistance strains possess inducible,high-level resistance to vancomycin(MICs $\geq 64 \mathrm{ug} / \mathrm{ml}$ ) and teicoplani(MICs $\geq 16 \quad \mu \mathrm{g} / \mathrm{ml}$ ) [22]Resistance can be induced by glycopeptides (vancomycin,teicoplanin,avopercin, and ristocetin and nonglycopeptide agents such as bacitracin,polymixinB, androbinidine, a drug used to treat coccidal infections in poultry [23]. The details of vancomycin resistance have been best documented with vanA gene cluster found on the transposon,or"jumping" genetic element,Tn1546[22].

\section{Resistance mechanisms and genetic classification}

Action of vancomycin on peptidoglycansynthesis. Under normal conditions of peptidoglycan synthesis in enterococci.two molecule of D-alanine are joined by a ligase enzyme to form D-Ala-Ala,which is then added to UPD-Nacetylymuramyl-tripeptide to form the UPD- $N$-acetylmuramyl-pentapeptide.TheUDP-Nacetylmuramyl-pentapeptide, when incorporated into the nascent peptidoglycan(transcosylation),permits the formation cross bridges(transpeptidation) and contributes to the strength of peptidoglycan layer[24].Vancomycin binds with high affinity to the D- Ala-D-Ala termini of the pentapeptide precurcor units, blocking their addition the growing peptidoglycan chain and preventing subsequent cross linking [25].

VanAglycopeptide resistance

The vanA gene and other genes involved in the regulation and expression of vancomycin resistance (vanR,vanH,vanX,andvanZ) are located on a10,581-bp transposon (Tn1546) of E.faceium, which often resides on a plasmid [26].Expression of these genes results in the synthesis of abnormal peptidoglycan precursors terminating in D-Ala-D-lactate instead of D-ALa-D-Ala.Vancomycin binds to D-Ala-D-Lac with marked lower affinity than it does to the normal dipeptide product [27]. The core protein functions favoring synthesis of pentadepsipeptide terminating in D-Ala-D-Lac are as follows.(a)VanA protein is a ligase of altered substrate specificity which produces D-Ala-D Lac in preference to D-Ala-D-Ala[28].(b)VanH protein is a D-hydroxy acid dehydrogenasewhich creates a poolof D-lactate for use in the above reaction [29].(c)VanX protein is a D,Ddipeptidase lacking activity against D-Ala-D-Lac.This enzyme reduces pools of D-Ala-D-Ala produced by the enterococcal ligase,thereby minimizing the competing synthesis of normal pentapeptide[25 rept].VanA alone cannot confer resistance to vancomycin,probably because D-hydroxy acids such as D-Lac are neither natural products present in the environment of enterococci nor produced by enterococci [30]. 
VanR and VAnS proteins constitute a two-component regulatory system that regulates the transcription of vanHAX gene cluster[31].VanS apparently functions as a sensor to detect the presence of vancomycin [22] or, more likely, some early effect of vancomycin on cell wall synthesis [32].VanS then signals VanR the response regulator , which results in activation, or turning on of the synthesis of some other proteins (VanH,VanA,andVanX) involved in resistance.InVanA phenotype strains, either vancomycin or teicoplanin can induce the transcription,but the precise signals are still unknown[33].vanY and vanZ may contribute to but are not essential for resistance.VanY protein is a D,D-carbopeptidase that cleaves the D-Ala terminal peptide from any normal peptide that may have been made ,contributing modestly to resisrance levels.VanZ modestly increases the MICs of teicoplanin but not vancomycin, though mechanisms that have not yet been elucidated.It is not essential to the expression of VanA pehenotype [34].Mapping of the vanA gene cluster from several U.S.isolates revealed some heterogenecity in organization [35,36].Tn1546 existed intact in some strains but had insertion-like sequences between vanS and $v a n H$ in others. These vancomycin resistance gene clusters may be incorporated into even larger mobile elements additional insertion like elements [35,36].

Cross-linking of the precursors to the growing peptidoglycan is processed in bacteria by the PBPs, with $\mathrm{PBP}_{5}$ being used in enterococci[37]. The replacement of D-Ala by D-Lac does not impair cross-linking of the modified precursors to the growing peptidoglycan chain .However ,PBPs other than $\mathrm{PBP}_{5}$, which are so far not known to play a role in cell wall synthesis, are probably required for processing the altered precursors [38].These high-molecular weight PBPs display a higher affinity for $\beta$-lactams.SinceVanA resistance is induceable, a shift in the PBPs occurs only in the presence of vancomycin and results in $\beta$-lactam hypersusceptibility. This effect explains the synergy displayed by the combination of the two classes of drugs against vancomycin-resistant strains [39].

VanBglycopeptide resistancein enterococci is mediated by an abnormal ligase (VanB) that is structurally related to $\operatorname{Van} A(76 \%$ amino acid identity).VanB protein also favors the production of the pentadepsipeptide terminating in D-Ala-D-Lac[40].Genes analogous to their class A resistance counterparts are designated $\operatorname{vanH}_{B}$, vanX $_{B}, \operatorname{van}_{B}, \operatorname{vanR}_{B}$, andvan $S_{B}[41]$.Levels of D,D-dipeptidase activity (vanXB) correlates with levels of vancomycin resistance[42].There is high degree of sequence identity(approximately 70\%)between vanHAX and vanHBBXB but considerably less homology( 25 to $35 \%$ sequence homology) between RS and Y proteins of VanA and VanBVRE[42].There is no gene counterpart of vanZ in these organisms.van $Y_{B}$ is not found in all strains, and its position in the gene clusters differs from that of vanYinTn1546[43].Recent reports have shown DNA sequence heterogeneity, suggesting three subtypes of vanB ligase gene:vanB-1,vanB-2.andvanB3[44].the regulatory system in class B strains appears insensitive to induction by teicoplanin [42,rept].Teicoplanin induces the synthesis of VanA-related proteins but does not induce the production ofVanB-related proteins. On the other hand,vancomycin induces the synthesis of the resistance proteins of both systems, and in fact, if a teicoplanin susceptible enterococcus with vanB gene cluster is preexposed to vancomycin,the strain then tests teicoplanin resistant as well. In addition,teicoplanin-resistant mutants can be derived from teicoplanin-susceptible, vanBcontaining enterococci when these organisms are plated onto teicoplanin containing agar. Such mutants can also arise in vivo during therapy [17].possible mechanisms for teicoplanin resistance of these mutants include the loss of their requirement for an inducer (that is ,if they constitutively produce high levels of the vancomycin resistance proteins) and the ability of teicoplanin to act as inducer. [39].

VanCglycopeptideresistance.Enterococcus strains i.e. E.gallinarum,E.casseliflavus and E.flavescenceexhibit low level resistance to vancomycin. The nucleotide sequences of the vanC-1 gene in E.gallinarum,the vanC-2 gene in E.casseliflavus , and the vanC-3 gene in E.flavescence have been reported, there is some disagreement about whether E.flavescence is a legitimate enterococcal species [45].VanC ligase of E.gallinarum favors the production of a pentapeptide terminating in D-Ala-D-Ser.Substitution of D-Ser for D-Ala is presumed to weaken the binding of vancomycin to the novelpentapeptide.Insertionalinactivation of vanC-lunmasks the concomitant production of the D-Ala-D-Alapentapeptide in E.gallinarum [46].D,D-Dipeptidase and D,Dcarboxypeptidase activities analogous to those VanAand VanB strains have been described.It is presumed that the level of resistance expressed represents the balance achieved between normal and abnormal peptidoglycan synthesis [47].

VanDglycopeptideresistance.A novel vancomycin resistance gene designated $v a n D$ was first described in a NewYork Hospital in 1991 [48].The strain carrying this resistance trait was an E.faceium strain that was inhibited by vancomycin at $64 \mu \mathrm{g} . \mathrm{ml}$ and teicoplanin at $4 \mu \mathrm{g} / \mathrm{ml}$.Partial sequencing of the ligase gene showed that it was distinct from but similar to the $v a n A$ and $v a n B g e n e s$. Recently three clinical isolates of vancomycin resistant E.faceium carrying the VanD resistance trait were found in Boston [49]. and the deduced amino acid sequence of VanD showed 67\% identity to those of VanA and VanB.VanD appears to be located on the chromosome and is not transferable to other enterococci [39]. 
VanEglycopeptideresistance.The vane vancomycin resistance gene has recently been described in E.faceium BM4405, which is resistant to low levels of vancomycin (MIC, $16 \mu \mathrm{g} / \mathrm{ml}$ ) and susceptible to teicoplanin (MIC0.5 $\mu \mathrm{g} / \mathrm{ml}$ ).[50].This new genotype has similarities to the intrinsic VanC type of resistance. The deduced amino acid sequence has a greater identity toVanC (55\%) than to $\operatorname{VanA}(45 \%)$ or $\operatorname{VanD}(44 \%)$.[50].Lactobacillus casei,Pedococcu spentosacus and Leuconostoc mesenteroides are naturally resistance to glycopeptides [51].

Vancomycin-dependent enterococci (VDE).An interesting phenomenon that has developed in some strains of VanA-and VanB type VRE is that of vancomycin dependence [52].These enterococci not just resistant to vancomycin but now require it for growth Vancomycin dependent enterococci have been recovered from apparently culture- negative clinical samples by plating them onto vancomycin-containing agar such as that used for Campylobacter or gonococci. A likely explanation for the phenomenon of vancomycin dependence is that these enterococci turn off their normal production of D-Ala-D-Ala and grow only if a substitute dipeptide-like structure is made [17].

\section{CONTROL OF VRE IN HELTHCARE SETTING}

Epidemiology and beginning of VRE strains spread in Europe and the United States have notable differences. In Europe,glycopeptide avoparcin was frequently fed to animals as a growth enhancer, apparently selecting for the VRE commonly found in the intestinal flora of animals. The contamination of food from animals, such as poultry products, presumably led to the VRE colonization seen in a number of healthy individuals from European countries. In the United States, on the other hand glycopeptides have never been approved for animal feed use and VRE carriage was largely undetected.(except the endogenously resistant species E.gallinarum and E.casseliflavus) outside health care setting, at least early on. The wide use of vancomycin in the hospital setting is likely one of the culprits for rapid selection and proliferation of VRE within this environment. The proportion of VRE among enterococcal isolates from nosocomial infections has continued to rise during recent years, although with a lower rate of increase, as reported by the Centers for Disease Control and prevention(CDC) [53].

\section{VRE transmission}

VRE transmission by health care workers whose hands become transiently contaminated with the organism while caring for infected patients probably the most common mode of nosocomial transmission. This concept is supported by the recovery of VRE and other resistant enterococci from cultures of specimens from hands of health care workers [54].VRE transmission may also occur by way of contaminated medical equipment, although this is probably much less important than transmission by the hands of personnel. Electronic thermometers contaminated with outbreak strain were epidemiologically implicated in an outbreak[55].The spread of VRE via bedpan washer machines has been reported(PR.Chadwicket al,Letter,Lancet 344:685,1994).Nosocomial transmission of VRE has been attributed to the use of fluidized microsphere bed from which multiply resistant strains were recovered despite repeated attempts at decontamination by the manufacturers [56].Enterococci have been recovered from 7 to $30 \%$ of environmental cultures during several outbreaks[57].Since enterococci remain viable for several days to weeks on dry surfaces, it seems plausible that contaminated surfaces may act as a source from which personnel may contaminate their hands or clothing [57].

Disposable cover gowns worn by personnel who care for VRE patients have also been shown to be contaminated with patient's organism [58].Presumably, the clothing of the personnel who do not wear cover gowns may also become contaminated with VRE. However, at present there is no conclusive proof that VRE are spread by contaminated clothing. There is no proof that enterococci, including VRE, are spread by the airborne route. Recovery of VRE from animal sources in parts of Europe suggests that food borne transmission my occur in certain geographic areas [59].

\section{Prevention and control of VRE}

The epidemiology of VRE has not been elucidated completely; however, certain patients populations are at increased risk for VRE infection or colonization. These include critically ill patients or those with severe underlying disease or immunosuppression, such as ICU patients or patients on oncology or transplantation wards, those who have had an intra-abdominal or cardiothoracic surgical procedures, those with an indwelling urinary or central venous catheter, and those who have had a prolonged hospital stay or received multiple antimicrobial agents [57].CDC, hospital Infection Control Practices Advisory Committee (HICPAC) published recommendations in February 1995 [60], These recommendations mainly focused on (i)prudent use of vancomycin,(ii)education of hospital staff,(iii)effective use of microbiology laboratory, and (iv) implementation of infection control measures. To minimize nosocomial transmission of VRE, hospitals must use multidisciplinary approach that requires participation by a variety of departments and personnel [60]. 
Restricted usage of cephalosporin has been associated with decreased VRE infection and transmission in hospitals [61].Antimicrobial stewardship (AMS) is fundamental in the control of major hospital pathogens. In particular, restriction of the use of extended- spectrum cephalosprins, quinolones is of proven worth for VRE, MRSA, MRGN and C.difficle [62].

Control measures recommended by HICPAC to prevent patient to -patient transmission of VRE include: (a) Place VRE-infected or-colonized patients in single rooms or in same room as other patients with VRE [57].(b)Wear nonsterile gloves when entering the room of a VRE -infected or-colonized patient. During the course of caring patient, a change of gloves me be necessary after contact with material that may contain high concentrations of VRE[57].(c)Wear a clean nonsterile gown when entering the room of a VRE-infected orcolonized patient if substantial contact with the patient or environmental surfaces in the patient's room is anticipated od if patient is incontinent or has diarrhea, an ileostomy,or wound drainage and contained by dressing [57] (d) Remove gloves and gowns before leaving the patient's room and wash hands immediately with an antiseptic soap or use waterless agent [63].Hands can be contaminated via glove leaks or during glove removal, and bland soap is relatively ineffective in removing VRE from the hands[63].(e)Ensure that after glove and gown removal and hand washing clothing and hands do not contact environmental surfaces potentially contaminated with VRE(e.g., door knob or curtain) in the patient's room [57].Although it is not recommended by HICPAC ,some hospitals require that gowns and gloves be worn routinely by all personnel entering VRE patient's room [57].Anecdotal experience in an ICU in which outbreaks caused by a single clone of VRE occurred revealed that routinely wearing gowns and gloves before entering the rooms of patients, combined with other infection control measures, was effective in terminating the outbreaks [57].Handwerger et. al. also reported that the use of gowns in addition to other infection control procedures terminated outbreaks [64].The HICPAC has some additional recommendations for hospitals with endemic VRE or continued VRE transmission despite the implementation measures [60].In 2005,Lactobacillusrhamnosus GG(LGG),a strain of L.rhamnosus, was used successfully for first time to treat gastrointestinal carriage of VRE in renal patients [65].

\section{CONCLUSION}

Transmission control of vancomycin resistant enterococci (VRE) Include CDC Hospital Infection Control Practices Advisory Committee (HICPAC) recommendations and restricted usage of vancomycin and cepahalosporin in the hospital.

\section{REFERENCES}

[1]. Utley AH,Collins CH,Naidoo J,etal.Vancomycin-resistant enterococci.Lancet1.1988;(8575-6):57-8.PMID2891921

[2]. LeclercqR,DerlotE,DuvalJ,CourvalinP.Plasmid- mediated resistance to vancomycin and teicoplanin in Enterococcus faceium.NEng J Med.1988;319(3):157-61.

[3]. HidronAI.EdwardsJR,PatelJ,etal."NHSN annual update: antimicrobial-resistant pathogens associated with healthcare -associated infections:annual summary of data reported to National Healthcare Safety Network at the the Centers for Disease Control and Prevention,2006-2007'.Infection Control HospEpidemiol .2008;29(11):996-1011.doi:10.1086/591861.

[4]. Harwood VJ,BrownellM,PerusekW, et al'.Vancomycin- resistant Enterococcus spp.isolated from wastewater and chicken feces in the United States"Appl EnvironMicrobiol.2001;67(10):4930-3.doi:10.1128/AEM.10.4930-4933.

[5]. Cox IA,Jr,PopkenDA."Quantifying human health risks from virginiamycin used in chikens"Risk Analysis.2004;24(1):271-88.

[6]. OrsiGB,CiorbaV.Vancomycin resistant enterococci healthcare associated infections.Ann Ig.2013;25:485-92.

[7]. Lederberg R,et al.Plasmid-mediated resistance tovancomycin and teicoplanin in Enterococcus faceium.NEng J Med.1988;329:157 161.doi: 10.1056/NEJM 198807213190307

[8]. Willem RJ,etal.Global spread of vancomycin -resistant Enterococcus faceium from distinct nosocomial genetic complex.Emerg Infect Dis.2005;11:821-28.doi:10.3201/1106041204

[9]. Johnson PD,et al. A sustained hospital outbreak of vancomycin-resistant Enterococcus faceium bacteremia due to emergence of vanBE.facium sequence type 203.J Infect Dis.2010;202:1278-86.doi:10.1086/656319.

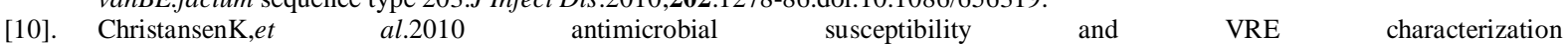
report.http://www.org/filesENTE\%202010\%20Report\%20Final\%20Secure pdf(accessed 14 January 2014 ).

[11]. HoshuyamaT,MoriguchiH,MurantiT,etal.Vancomycin-resistant enterococci(VRE) outbreak at a university hospital inKitakyushu,Japan:case control studies.J InfectChemother.2008;14(5):354-60.doi:10.1007/s10156-008-0628.

[12]. Mutters NT,etal.Control of the spread of vancomycin-resistant Enterococci in hospital:epidemiology and clinical relevance.DischArztebl Int.2013;110:725-31.

[13]. McbrideSJ,etal.Clinical characteristics and outcomes of patients with vancomycin susceptible Enterococcus faecalis and Enterococcus faceiumbacteraemia:five year retrospective review.EurJClinMicrobiol Infect Dis.2010;29:107114.doi:10.1007/s10096-009-0830-5.

[14]. CourvalinP.Vancomycin resistance in gram-positive cocci.Clin Infect Dis.2006; 42:S25-S34.

[15]. WilemsRJ,BontenMJ.Glycopeptide-resistant enterococci:deciphering virulence, resistance and epidemicity.CurrOpin Infect Dis.2007;20:384-390.

[16]. Murray BE. The life and times of the enterococcus.ClinMicrobiol Rev.1990;3:45-65

[17]. Murray BE, Vancomycin resistant enterococci.Am J Med.1977;101:284-293.

[18]. FontanaR,BertoliniG,AmalfitanoG,CanepariP.Characterization of penicillin resistant Streptococcocus faceiummutants .FEMSMicrobiol Lett.1984;25:21-25. 
[19]. Fontana R,GrossataA,Rossi,etal.Transition from resistance to hypersusceptibility to $\beta$-lactam antibiotics associated with loss of a lower-affinity penicillin binding protein binding in Streptococcous facium mutant highly resistant to penicillin.Animicrob Agent Chemother.1985;28:678-683.

[20]. Herman DJ,Gerding DN. Screening and treatment of infections caused by resistant enterococci .Antimicrob Agents Chemother.1991;35:215-219

[21]. MoelleringRC,Jr,WeinbergAN.Studies on antibiotic synergism against enterococci.II.Effect of various antibiotics on update of 14Clabelled streptomycin by enterococci.J.Clin Investig.1971;50:2580-84.

[22]. Arthur M,CourvallinP.Genetics and mechanisms of glycopeptide resistance in enterococci.Antimicrob Agents Chemother.1993;37:1563-71.

[23]. Lai MH,Kirsch DR.Induction signals for vancomycin resistance encoded by the vanAgene cluster in Enterococcus faecium.Antimicrob Agents Chemother.1996;40:1645-48.

[24]. EliopoulusGM,Vancomycin-resistant enterococci:mechanism and clinical relevance.Infect Dis Clin North Am.1997;11:851-65.

[25]. Wu Z,WrightGD,WalshCT.Overexpression,purification and characterization of van X,a D-D-dipeptide which is essential for vancomycin resistance in Enterococcus faecium BM4147.Biochemistry.1995;34:2455-63.

[26]. Arthur M,MolinasC,DepardienF,etal.Characterization of Tn1546, aTn3-related transposon conferring glycopeptide resistance by synthesis of desipeptide peptidoglycan precursors in Enterococcus faecium.BM4147.J Bacteriol. 1993; 175:117-127.

[27]. BuggTDH,WrightGD,Dutka-Malen, etal.Molecular basis for vancomycin resistance in Enterococcus faecium BM4147:biosynthesis of a despeptide peptidoglycan precursor by vancomycin resistance proteins VanH and vanA.Biochemistry.1991;30:1408-15.

[28]. BuggTDH,Dutka-MalenS,ArthurM,etal.Identification of vancomycin resistance protein VanA as a D-alanine-S-alanine ligase of altered substrate specificity.Biochemistry.1991;30:2017-21.

[29]. Arthur M, MolinasC,Dutka-Malen,etal.Structural relationship between the vancomycin resistance protein VanH and 2hyroxycarboxylic acid dehydrogenases. Gene.1991;103:133-34.

[30]. LeclereqR,CourvalinP.Resistance to glycopeptides in enterococci.Clin Infect Dis.1997;24:545-556.

[31]. Arthur M,DepardieuF,Gerbaud G,et al.The vanS sensor negatively controls vanR-mediated transcriptional activation of glycopeptide resistance gene of Tn1546 and related elements in absence of induction.J Bacteriol.1997;179:97-106.

[32]. HandwergerS,KolokathisA.Induction of vancomycin resistance in Enterococcusfaecium by inhibition of transglycosylation.FEMSMicrobiol Lett.1990;58:167-70.

[33]. BaptistaM,DepardieuF,CourvalinP,etal.Specificity of induction of glycopeptide resistance in Enterococcus faecalis.Antimicrob Agents Chemother.1996;40:2291-2295.

[34]. ClevelDB,Movable genetic elements and antibiotic resistance in enterococci.Eru JClinMicrobiol Infect Dis.1990;9:90-102.

[35]. HandwergerS,SkobeJ,DiscottoI,etal.Heterogenecity of the vanA in clinical isolates of enterococci from the Northeastern United States .Antimicrob AgentsChemother.1995;39:362-68.

[36]. HandwergerS,Skoble J.Identification of chromosomal mobile element conferring high level vancomycin resistance in Enterococcus faecium.Antimicrobiol Agents Chemother.1995;39:2446-53.

[37]. Fontana R,BertoliniG,AmalfitanoG,etal.Characterization of penicillin-resistant Streptococcus faeciummutants.FEMSMicrobiol Lett.1984;25:21-25.

[38]. AI-Obeid S, Billot-Klein D,vanHeijeoortJ,etal.Replacement of the essential penicillin -binding protein 5 by high molecule mass PBPs may explain vancomycin $\beta$-lactam synergy in low level vancomycin- resistant Enterococcus faecium D366.FEMS Microbiol Lett.1992;79-84.

[39]. YesimCetinkaya,PamelaFalk,C.GlenMayhall.Vancomycin-Resistant Enterococci. Clin.Microbiol Rev.2000;13(4):688-707

[40]. Evers,SahmDF,Courvallin P.The van B gene of Enterococcus faecalis v583.is structurally related to genes encoding D-Ala,D-Ala ligases and glycopeptide -resistance proteins vanA and vanC.Gene.1993;124:143-144

[41]. Caron F,Carbon C, GutmannI.Triple combination of penicillin-vancomycin-gentamycin for experimental endocarditis caused by a moderately penicillin-and highly glycopeptide-resistant isolates of Enterococcus faecium.J InfectDis.1994;164:888-93.

[42]. Arthur M,DepardieuF,ReynoldP,etal.Quantitative analysis of the metabolism of soluble cytoplasmic peptidoglycan precursors of glycopeptide resistance enterococci.Mol Microbiol.1996;21:33-44.

[43]. Evers S, CourvalinP.Regulation of Van B type vancomycin resistance gene expression by the VanS ${ }_{B}-V_{a n R_{B}} t_{w o}$ componentregulatory system in Enterococcus faecalis V583.J Bacteriol.1996;178:1302-1309.

[44]. Dahl KH,SimonsenGS,Olsvik $\emptyset_{2}$ et al.Heterogenety in the vanB gene cluster of genomically diverse clinical strains of vancomycinresistant enterococci.Antimicrob Agents Chemother.1999;43:1105-1110.

[45]. Clark NC,TeixeriaIM,FacklamRR,etal.Detection and differentiation of vanC-1,vanC-2, and vanC-3 glycopeptide resistance gene in enterococci.JClinMicrobiol. 1998 ;36: 2294-2297.

[46]. Reynolds PE,SnaithHE,MaguireAJ,etal.Analysis of peptidoglycan precursors in vancomycin resistant E. gallinarumBM4174.Biochem J.1994;301:5-8.

[47]. Eliopoulos GM,Vancomycin-resistant enterococci mechanism and clinical relevance. Infect Dis North Am.1997;11:851-65.

[48]. PerichonB,ReynoldsP,CourvalinP.VanD type glycopeptide-resistant Enterococcus faecium BM4339.Antimicrob Agents Chemother.1997;41:2016-18.

[49]. OstrowskyBE,ClarkNC,Thuvin Eliopoulos C,etal.A cluster of vanD vancomycin-resistant Enterococcus faecium: molecular characterization and clinical epidemiology.J Infect Dis.1999;180:1177-85

[50]. Fine M,PerichonB,ReynoldsP,etal.VanE,a new type of acquired glycopeptide resistance in Enterococcus faecalis.BM4405.Antimicrob Agents Chemother. 1999; 43:2161-64.

[51]. Billot-Klein D,GutmannI,SableS, etal.Modification of peptidoglycan precursors in a common feature of the low-level vancomycinresistant VanB type enterococcus D366 and of the naturally glycopeptide-resistant species Lactobacillus casei,Pseudomonaspentosaceus,Leuconostocmesenteroides, andEnterococcus gallinarum. J.Bacteriol. 1994;176:2398-2405.

[52]. DeverLL,Smith SM,HandwergerS,etal.Vancomycin-dependent Enterococcus faceium isolated from stool following oral vancomycintherapy.JClinMicrobiol. 1995;33:2770-73.

[53]. National Nosocomial Infections Surveillance (NNIS) System Report, data summary from January 1992,through June 2004, issued October 2004.Am J Infectcontrol.2004;32:470-85.

[54]. TornieporthNG,Roberts RB, John J,et al. Risk factors associated with vancomycin resistant Enterococcus faecium infection or colonization in 145 matched case patients and control patients .Clin Infect Dis.1996;23:767-72.

[55]. Livornese LL, Dias s, SamelC,etal.Hospital acquired infection with vancomycin-resistant Enterococcus faecium by electronic thermometers.Ann Internmed.1992;117:112-116.

[56]. Freeman R, Gould FK, Ryan DW,etal.Nosocomial infection due to a fluidized microsphere bed. The value of prophylaxis mass spectrometry.J Hosp Infect.1994;27:187-193. 
[57]. Boyce JM,OpalSM,ChowJW,etal.Outbreak of multi-drug resistant enterococcus faecium with transferable VanB class vancomycinresistance.JClinMicriobiol.1994;32:1148-1153.

[58]. Boyce Jm,MermelLA,ZervosMJ,et al.Controlling vancomycin resistant enterococci.Infect Control Hosp Epidemiol.1995;16:634637.

[59]. White W,KlareI.Glycopeptide-resistant Enterococcus faecium outside the hospitals: a commentary.Microb Drug Resist.1995;8:259263.

[60]. Centers for Disease Control and Prevention. Recommendations for preventing spread of vancomycin resistant. Infect Control Hosp.1995;16:105-113

[61]. ClaversIS,MoserSA,BenjaminWH,etal.Vancomycin -resistant enterococci:15 years and counting .J Hosp Infect.2003;53(3):159-71

[62]. Dance S.Approaching zero: temporal effects of a restrictive antibiotic policy on hospital acquired Clostridium defficle, extended spectrum $\beta$-lactamase producing coliforms and methicillin Staphylococcus aureus.Int J AntimicrobAgents. 2013;41:137142.doi:10.1016/ijanticag,201210.013.

[63]. Doebbling BN, Stanley GI,SheetzCT,etal.Comparative efficacy of alternative hand-washing agents reducing nosocomial infections in intensive care units.NEngl JMed.1992;327:88-93.

[64]. HandwegerS, RaucherB,AltaracD,etal.Nosocomial outbreak due to Enterococcus faecium highly resistant vancomycin,penicillin,and gentamicin,Clin InfectDis.1993;16:750-755.

[65]. Manley KJ,FraenkleMB,MayallBC,etal. Probiotic treatment of vancomycin resistant enterococci:a randomized controlled trial.Med J Aus.2007;186 (8):454-7. 